\title{
APOYO ORGANIZACIONAL PERCIBIDO Y COMPROMISO ORGANIZACIONAL, SU RELACIÓN CON EL CLIMA ÉTICO EN ENTIDADES DE FISCALIZACIÓN SUPERIOR
}

\section{PERCEIVED ORGANIZATIONAL SUPPORT AND ORGANIZATIONAL COMMITMENT, IT'S RELATIONSHIP WITH THE ETHICAL CLIMATE IN SUPREME AUDIT INSTITUTIONS}

Tomás Francisco Morales Cárdenas*, Abel Pérez Sánchez**, Erik Haidar Torres***

\begin{abstract}
*Doctorante en Administración. Universidad Autónoma de Guadalajara Campus Tabasco Av. Prolongación Paseo Usumacinta Km 3.5 S/N Ra. Emiliano Zapata, Colonia El Country, Villahermosa, Tabasco, C.P. 61107. Teléfono de contacto: 9933112952. ORCID: https://orcid.org/0000-0002-5934-2748.

**Doctor en Administración. Universidad Autónoma de Guadalajara, Campus Tabasco. Email: aperezs@uagtabasco.edu.mx. ORCID: https://orcid.org/0000-0002-0088-1122.

***Doctor en Administración. Email: erickhaidart@gmail.com. ORCID: https://orcid.org/0000-0002-58564736.
\end{abstract}

Dirección para recibir correspondencia: tomcard@hotmail.com 
APOYO ORGANIZACIONAL PERCIBIDO Y COMPROMISO ORGANIZACIONAL, SU RELACIÓN CON EL CLIMA ÉTICO EN ENTIDADES DE FISCALIZACIÓN SUPERIOR

\section{RESUMEN}

OBJETIVO: Estudiar la apreciación del Apoyo Organizacional Percibido (AOP), el Compromiso Organizacional (CO) y sus relaciones con el Clima Ético (CE), en una muestra de empleados en siete Entidades de Fiscalización Superior de las Legislaturas Locales (EFSLL) en México.

MATERIAL Y MÉTODO: La investigación tiene enfoque cuantitativo, correlacional confirmatorio; la muestra fue aleatoria de 250 empleados de un universo de 1,644 que laboran en siete EFSLL participantes. Se describen las características de las teorías de los constructos de la investigación para el desarrollo del modelo de investigación, AOP, CO y CE. El instrumento se conformó de 65 ítems en escala Likert de siete puntos y fue aplicado en línea. El análisis confirmatorio se efectuó mediante modelado de ecuaciones estructurales, para integrar y comprobar el modelo teórico se analizaron las subdimensiones de CO y CE, así como del constructo unidimensional AOP, hasta obtener un buen ajuste en cada uno de ellos y del modelo global.

RESULTADOS: Los resultados permiten afirmar la comprobación de las hipótesis del estudio donde el AOP y el CO se relacionan positivamente e impactan al CE. El peso de regresión estandarizado de AOP tiene un efecto positivo sobre CO la relación positiva es de 0.756 que confirma la hipótesis de estudio; el peso de regresión estandarizado de CO tiene un efecto positivo sobre CE la relación positiva es de 0.187 que confirma la hipótesis de estudio; el peso de regresión estandarizado de AOP tiene un efecto positivo sobre CE la relación positiva es de 0.732 que confirma la hipótesis de estudio.

CONCLUSIONES: Los servidores públicos de los EFSLL que conformaron la muestra, tienen moderado compromiso de lealtad con la institución, sus lazos afectivos aún están en desarrollo, la permanencia en sus trabajos no es de consideración debido a las características especializadas del trabajo que desempeñan. Las personas pueden sentir de manera simultánea y distinta intensidad, más de un tipo de compromiso debido a que son el resultado de las experiencias personales, que atienden particularidades de la organización, se determinó la presencia de los tres tipos de climas éticos; la influencia del AOP es significativa esto es, ante una percepción de mayor apoyo de la organización, se estimula directamente el CEB y el CEE e indirectamente el de principios.

PALABRAS CLAVE: Compromiso organizacional. Apoyo organizacional. Clima ético. Entidades de fiscalización superior. 
APOYO ORGANIZACIONAL PERCIBIDO Y COMPROMISO ORGANIZACIONAL, SU RELACIÓN CON EL CLIMA ÉTICO EN ENTIDADES DE FISCALIZACIÓN SUPERIOR

\section{ABSTRACT}

OBJECTIVE: To study the appreciation of the Perceived Organizational Support (POS), the Organizational Commitment $(O C)$ and its relationships with the Ethical Climate (EC), in a sample of employees in seven Supreme Audit Institutions of the Local Legislatures (SAILL) in Mexico.

MATERIAL AND METHOD: This research has a quantitative, correlational and confirmatory approach; the sample was randomized from 250 employees from a universe of 1,644 who work in seven SAILL participants. The characteristics of the theories of the research constructs for the development of the research model, POS, OC and EC are described. The instrument was formed by 65 items on a seven-point Likert scale and was undertaken online. The confirmatory analysis was made by modeling structural equations, to integrate and check the theoretical model, the subdimensions of the $O C$ and the $E C$, as well as the one-dimensional construct POS were analyzed, until a good adjustment in each of them and in the global model was obtained.

CONCLUSIONS: The civil servants of the SAILL who participated in the sample have a moderate commitment of loyalty to the institution, their emotional ties are still developing, the permanence in their jobs is not relevant due to the specialized characteristics of the kind of work they perform. People can feel simultaneously and with different intensity, more than one type of commitment as the result of personal experiences which address the particularities of the organization, the presence of the three types of ethical climates was determined; The influence of the POS is significant, that is, before the perception of greater support from the organization, the BEC and the EEC are indirectly stimulated, and indirectly, the one regarding the principles.

KEY WORDS: Organizational commitment. Organizational support. Ethical climate. Supreme audit institutions.

\section{INTRODUCCIÓN}

"El resultado futuro de cada organización está en función de la gestión de sus recursos humanos, con medidas dirigidas a desarrollar un compromiso de carácter afectivo para permitir retener el talento de sus empleados, debido al vínculo entre el compromiso con la organización y la actitud, el comportamiento y los resultados de sus miembros" (Pons y Ramos, 2010). 
APOYO ORGANIZACIONAL PERCIBIDO Y COMPROMISO ORGANIZACIONAL, SU RELACIÓN CON EL CLIMA ÉTICO EN ENTIDADES DE FISCALIZACIÓN SUPERIOR

De acuerdo con los resultados 2017 de la Encuesta Nacional de Calidad e Impacto Gubernamental (ENCIG), en el año 2017 la tasa de prevalencia de actos de corrupción en trámites gubernamentales ascendió a $14.6 \%$, un crecimiento de dos puntos porcentuales más que en 2015 (INEGI, 2018).

De acuerdo con Robbins y Judge (2013), la intención de comportarse de cierta forma hacia alguien o algo; deben considerarse elementos como la cognición, afecto y comportamiento. Lo anterior, ha originado investigaciones en el ámbito del comportamiento organizacional, con el correspondiente desarrollo de modelos, teoría e instrumentos donde se fundamentan, explican, miden y también cuestionan cada constructo y sus relaciones. Dentro de estas actitudes destacan el Apoyo Organizacional Percibido (AOP), el Compromiso Organizacional (CO) y el Clima Ético (CE).

En las Entidades de Fiscalización Superior de las Legislaturas Locales (EFSLL), la operación asume los costos de la curva de aprendizaje de los nuevos colaboradores en la organización incluso de sus titulares donde la rotación de éstos que puede darse por separación voluntaria o involuntaria del cargo o por finalización de periodos de designación. En este sentido se llega al extremo de afirmar que funcionan con, sin o a pesar del titular, constituyendo una manifestación de compromiso hacia la organización y la existencia de procesos de trabajo normados y con plazos definidos. Sin embargo pueden presentarse situaciones que manifiesten debilidades en el clima ético y a su vez debilitan la confianza y credibilidad en sus actuaciones y resultados.

Por lo anterior el objetivo de la investigación fue determinar el impacto del AOP y el CO en el CE de las EFSLL. Los objetivos secundarios se dirigen a explicar las relaciones entre las dimensiones del $\mathrm{CO}$ y el AOP, así como explicar cómo las dimensiones del CO y el AOP impactan en las dimensiones del CE (ver figura 1). 
APOYO ORGANIZACIONAL PERCIBIDO Y COMPROMISO ORGANIZACIONAL, SU RELACIÓN CON EL CLIMA ÉTICO EN ENTIDADES DE FISCALIZACIÓN SUPERIOR

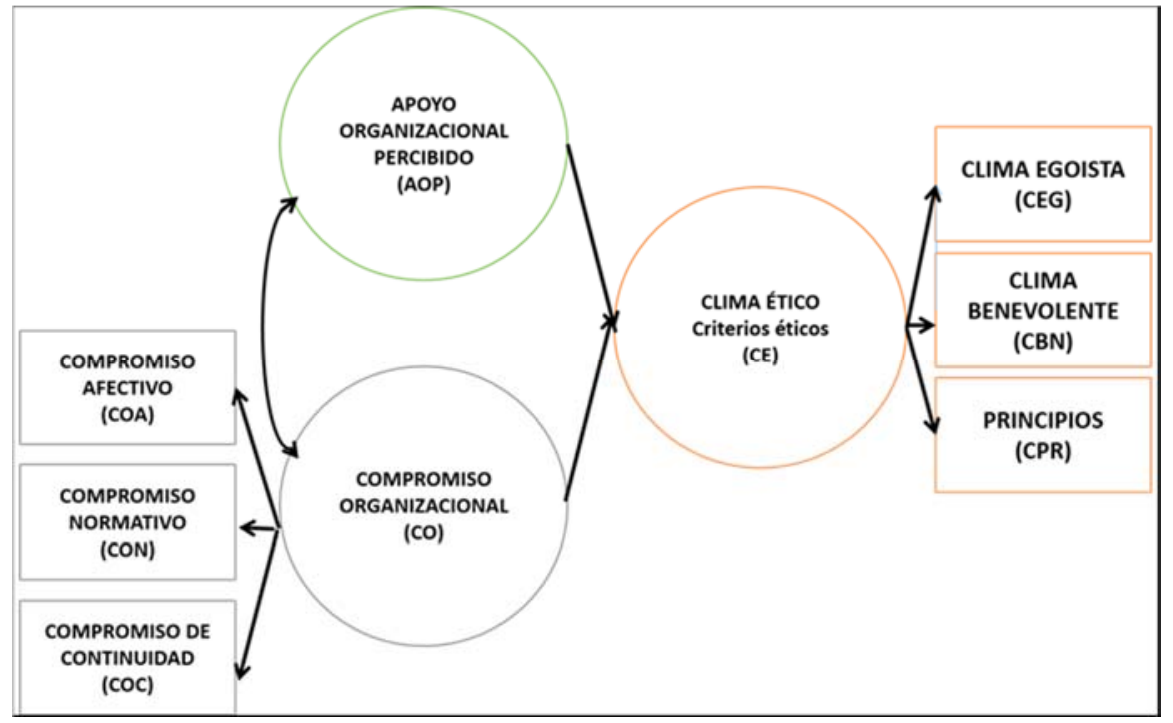

\section{Figura 1. Modelo propuesto para la investigación.}

Fuente: Elaboración propia con base en los modelos e instrumentos de Einsenberger, Huntingon y Sowa (1986); Allen y Meyer (1990); Victor y Cullen (1987).

Se puntualizan los alcances de esta investigación, mediante la formulación de hipótesis resultantes de las relaciones entre las variables sujetas a estudio; $\mathrm{H} 1$ : El CO y el AOP percibido se relacionan positivamente; $\mathrm{H} 2$ : El CO tiene efecto significativo en el CE de la organización y H3: El AOP tiene efecto significativo en el CE de la organización.

La teoría del intercambio social propone que los individuos se sienten obligados a retribuir el trato y apoyo que perciben de parte de la organización, explica la medida de las actitudes laborales y el comportamiento de los empleados. El comportamiento social es un intercambio de bienes tanto materiales como intangibles, representado en manifestaciones de símbolos de aprobación y prestigio, de esta forma las personas que más aportan a otras esperan obtener también mucho de ellas y las que obtienen mucho de otros tienen la presión de corresponder en la misma medida. Por ello se debe tener en cuenta que la norma de reciprocidad es aplicable no sólo entre personas sino también entre el empleado y la entidad para la que trabaja (Homans, 1958; Rousseau, 1989).

Cuando la acción de una persona recibe la recompensa que espera, se sentirá complacida y es más probable que realice la conducta aprobada para representar resultados valiosos; en el contexto del compromiso organizacional, se considera que los empleados intercambian su implicación con la organización por recompensas y beneficios; los empleados estarán 
APOYO ORGANIZACIONAL PERCIBIDO Y COMPROMISO ORGANIZACIONAL, SU RELACIÓN CON EL CLIMA ÉTICO EN ENTIDADES DE FISCALIZACIÓN SUPERIOR

comprometidos en la medida en que sus necesidades y expectativas se vean satisfechas en estas relaciones de intercambio (Álvarez, 2008).

La conducta de las personas en el trabajo depende, en gran medida de las percepciones de las dimensiones relevantes de las organizaciones por sus miembros (Topa y Moriano, 2008). Por esa razón, las organizaciones asumen actitudes asertivas, para crear un ambiente favorable en sus integrantes, donde el elemento humano es el eje fundamental (Torres y Díaz, 2012). El comportamiento de los empleados a menudo, depende de factores organizativos, como la cultura o el apoyo del líder, los empleados pueden voluntariamente aportar más allá de sus deberes laborales normales si confían en la organización (Duffy y Lilly, 2013).

El concepto AOP tuvo sus inicios en las observaciones y estudios realizados con la finalidad de corroborar el efecto de prácticas empresariales de una organización en el grado de compromiso de los empleados, lo que les permitió desarrollar la teoría de apoyo organizacional que se conceptualiza como la creencia de los empleados respecto del grado, en el cual la organización valora sus contribuciones y se preocupa por su bienestar.

El AOP contribuye a la explicación del compromiso organizacional afectivo, destaca los focos de identificación a disposición de las personas en el trabajo, tienen influencia sobre los resultados (Kirana et al. 2012, Miao, 2011). De acuerdo a la norma de reciprocidad, la relación entre AOP y CO funciona como una retroalimentación entre empleado-organización, bajo la premisa de que, si un trabajador se siente apoyado por su empresa, responderá comprometiéndose con ella, de allí que el AOP y el CO se correlacionen positivamente (Rhoades y Eisenberguer, 2002).

De acuerdo al CO, una persona se compromete con su organización por tres razones esenciales: se siente ligado emocionalmente a ella, percibe costos asociados ante la posibilidad de dejar la organización o al sentir que tiene una obligación moral de permanecer en ella, de allí que se considere como un estado psicológico de tres componentes que pueden ser separables e interrelacionados y es a lo que se denomina Compromiso Afectivo (CA), Compromiso Continuo (CC) y Compromiso Normativo (CN) (Allen y Meyer, 1990; Meyer y Allen, 1991).

Para los empleados, el CO representa estabilidad laboral, con impacto en prestaciones, jubilación, beneficios sociales y garantiza poder cubrir necesidades materiales y psicológicas como el reconocimiento, desarrollo de habilidades, socialización principalmente, con repercusión en el beneficio de las familias y economía del país. Para los responsables de la gestión de los 
APOYO ORGANIZACIONAL PERCIBIDO Y COMPROMISO ORGANIZACIONAL, SU RELACIÓN CON EL CLIMA ÉTICO EN ENTIDADES DE FISCALIZACIÓN SUPERIOR

recursos humanos, así como para los investigadores, la importancia del compromiso organizacional obedece en forma primordial al impacto directo en actitudes y conductas del empleado, reflejadas en la aceptación de metas, valores y cultura de la organización, de igual forma un menor ausentismo y baja rotación de personal (Betanzos y Paz, 2007).

EI CE se origina con las investigaciones de Víctor y Cullen, (1988) quienes presentaron una de integración de los conceptos de la literatura de clima de trabajo y la literatura del ambiente moral, para crear un marco para la investigación de los climas éticos en el trabajo. Combinaron las ideas de la atmósfera moral con los de clima laboral para producir el trabajo del marco e clima ético; desarrollaron una tipología de dos dimensiones, para investigar los patrones de toma de decisiones de los miembros de la organización (Webber, 2007); el cuestionamiento como constructo se debe a que una corriente lo considera como una subcategoría de clima organizacional o de trabajo (Arnaud y Schminke, 2006; Blome y Paulraj, 2013).

Por su parte, Víctor y Cullen (1987, 1988); Cullen y Bronson (1993) afirman que los responsables de las acciones éticas o no éticas que tienen lugar entre sus empleados son las propias organizaciones, en consecuencia, puede iniciar y poner en práctica los climas de trabajo éticos.

En la tipología teórica del CE se identifican dos dimensiones: Criterio ético, utilizado en la toma de decisiones de la organización, y el centro de análisis, que se refiere a la toma de decisiones éticas. De acuerdo con Víctor y Cullen, (1988). El criterio ético comprende los principios de comportamiento con los cuales la organización resuelve los dilemas morales como son, el egoísmo, la benevolencia, y los principios (Gómez y Manrique, 2014). El egoísmo corresponde a la maximizar el interés propio independientemente de situaciones opuestas de la sociedad o las opiniones de otras personas. La benevolencia es aquella donde la gente tiende a ser menos conscientes de las leyes y las normas y también pueden ser susceptibles a los argumentos que emplean reglas o principios. Los principios son otra dimensión del criterio ético, donde la gente es proclive a ser menos sensibles a los efectos particulares sobre los demás.

Por otra parte, el centro de análisis representa las diferentes fuentes de influencias y la motivación en que una persona podría concebir sus propias percepciones sobre cuestiones éticas o no éticas. Las categorías que comprenden esta dimensión son: individual, local, y cosmopolita. El individuo que se manifiesta en lo individual, encuentra la fuente de razonamiento en sí mismo; el conjunto de individuos de una organización, encuentran la fuente de razonamiento fuera de sí mismos expresado en lo local o finalmente, en la organización expresado en un agente del 
APOYO ORGANIZACIONAL PERCIBIDO Y COMPROMISO ORGANIZACIONAL, SU RELACIÓN CON EL CLIMA ÉTICO EN ENTIDADES DE FISCALIZACIÓN SUPERIOR

contexto social y fuera de la organización, que se refleja en lo cosmopolita (Ferrato y Coutinho, 2005; Gómez y Manrique, 2014). De acuerdo a lo anterior, la descripción del razonamiento moral de un empleado o individuo se obtiene al combinar las dos dimensiones: criterio ético y centro de análisis que forman nueve criterios diferentes.

La razón por la cual las EFSLL son es contexto de esta investigación obedece a que son las instituciones gubernamentales encargadas del proceso de fiscalización superior y por tanto requiere de una actuación ética de sus integrantes en su función de comprobar que la actividad económico-financiera de los entes integrantes del sector público para dictaminar sobre su adecuado ejercicio (Maher, 2004). El principal cuestionamiento a estas organizaciones es el valor y beneficio a la sociedad por su efectividad en el combate a la corrupción en las administraciones públicas (Rankumar, 2007).

\section{MATERIAL Y MÉTODO}

El enfoque de esta investigación es cuantitativo, correlacional y confirmatorio. La muestra de 250 servidores públicos se obtuvo de forma aleatoria de un universo de 1,644 que laboran en siete de los 32 EFSLL del país en áreas de fiscalización operativas, mandos medios y superiores que aceptaron participar, en ella quedaron representadas las cuatro regiones geográficas en que la Asociación Nacional de Organismos de Fiscalización y Control Gubernamental (ASOFIS) tiene clasificado el país. El nivel de confianza fue de1.96 con un error estimado del $5.7 \%$.

El instrumento utilizado para medir el AOP es la versión corta integrada por ocho ítems (Eisenberger, et al. 1986; Eisenberger, et al. 2002; Eisenberger, Fasolo y Davis-LaMastro, 1990); es unidimensional y tiene alta confiabilidad interna (Rhoades y Eisenberger, 2002). La escala de medición del CO utilizada es el cuestionario con 18 afirmaciones (Meyer, Allen y Smith, 1993) deriva del modelo tridimensional de este constructo. Para medir el CE se aplicó el cuestionario de treinta y seis preguntas (Cullen, et al. 1993; Victor y Cullen, 1987; Victor y Cullen, 1988), (ver tabla 1). 
APOYO ORGANIZACIONAL PERCIBIDO Y COMPROMISO ORGANIZACIONAL, SU RELACIÓN CON EL CLIMA ÉTICO EN ENTIDADES DE FISCALIZACIÓN SUPERIOR

\section{Tabla 1}

Operacionalización de variables y número de ítems del instrumento integrado

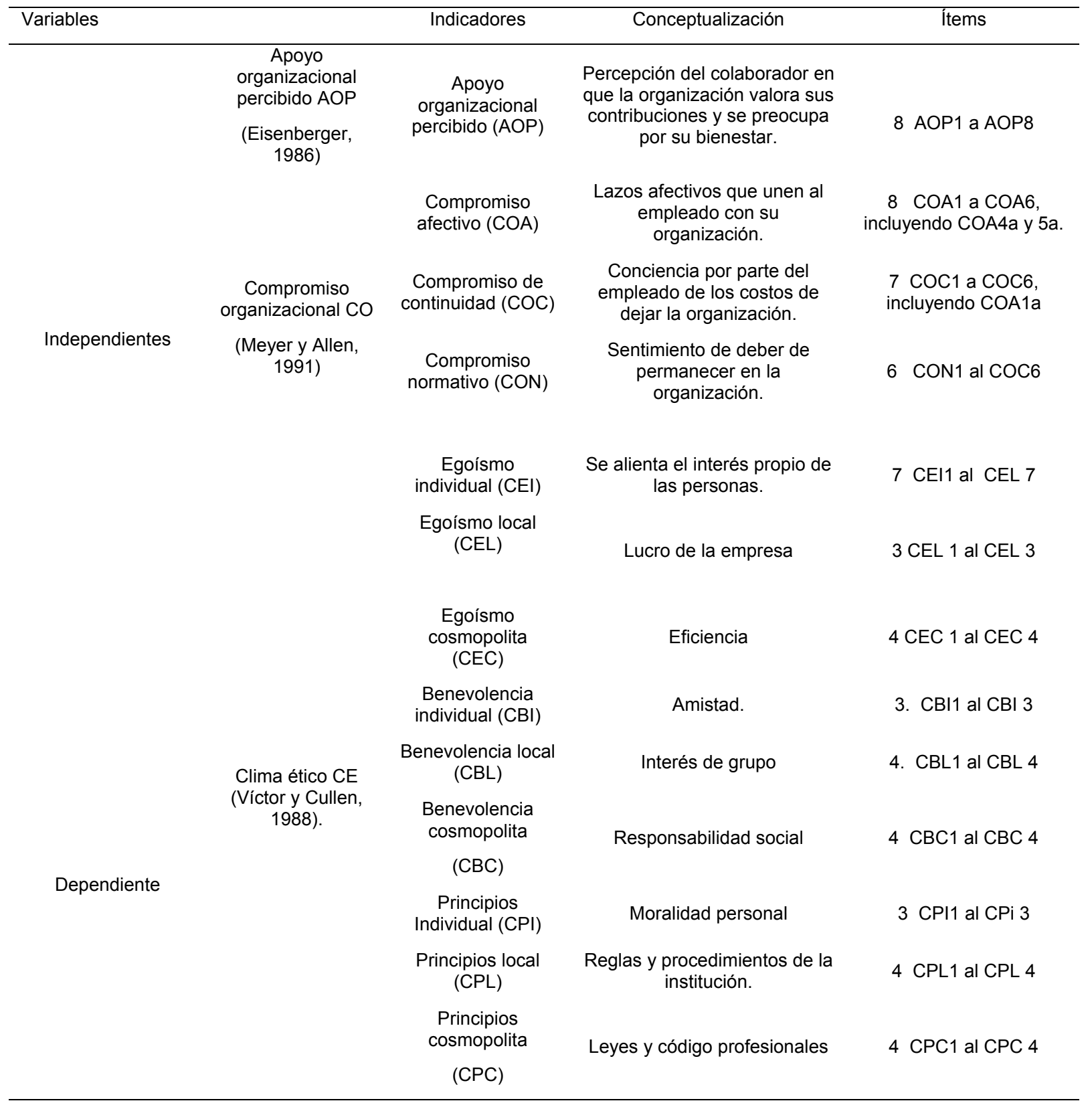

Fuente: Elaborado con base en instrumentos diseñados por Eisenberger, Huntingon y Sowa (1986); Allen y Meyer (1990); Víctor y Cullen (1988). 
APOYO ORGANIZACIONAL PERCIBIDO Y COMPROMISO ORGANIZACIONAL, SU RELACIÓN CON EL CLIMA ÉTICO EN ENTIDADES DE FISCALIZACIÓN SUPERIOR

\section{RESULTADOS}

En los resultados de la caracterización de los participantes se destaca una participación ligeramente mayor del género femenino, con 123 hombres $49.2 \%$ y 127 mujeres 50.8\%. La distribución por el nivel de estudio refleja un alto nivel de preparación profesional con carreras como: Contaduría 47.2\%, Administración 14.8\%, Ingeniería 14\%, Derecho $10.4 \%$, todas ellas relacionadas con las actividades de los EFSLL como es la auditoría gubernamental.

Las características sociodemográficas en relación al trabajo desarrollado por los participantes muestran que los participantes son principalmente personal operativo $64 \%$, donde el $46 \%$ manifestó tener personal bajo su mando y el $81.6 \%$ está contratado de forma permanente, solo el $7.2 \%$ esta sindicalizado; resalta el hecho de que el $40.8 \%$ cuentan con más de 10 años de servicio. Lo anterior, refleja la estabilidad de este tipo de empleos, donde es un requerimiento legal la existencia de un sistema de servicio civil de carrera.

Los parámetros de asimetría y curtosis para la evaluación de la normalidad multivalente de los ítems, se considera dentro de los parámetros aceptables. Para examinar la fuerza y la dirección de la relación lineal entre ítems del modelo se determinó el coeficiente de correlación de Pearson. Se destacan correlaciones de los cinco ítems de la variable AOP, donde salvo la correlación entre AOP1 y AOP7 con valor de 0.51 los restantes muestran fuertes correlaciones con valores entre 0.6 y 0.8. Los resultados del análisis de los siete ítems de las subdimensiones de la variable CO, muestran una fuerte correlación entre COA3 y COA 4a con un valor de 0.62 y la más débil es de 0.32 entre COA4 y CON5. En los resultados de los 12 ítems de las subdimensiones de la variable $\mathrm{CE}$, se observa una fuerte correlación entre CPC3 y CPL1 con un valor de 0.65 y las más débiles de 0.32 entre CPC3 con CPL4 y CBL2.

Se aplicó la prueba de Levene con la finalidad de realizar al análisis de homogeneidad de varianzas e identificar la existencia de homocedasticidad de acuerdo a los resultados obtenidos se asumen varianzas iguales. El resultado muestra índices de tolerancia con valores mayores a 0.1 , por lo que se rechaza la existencia de colinealidad entre los ítems, en los Factores de Inflación de la Varianza (VIF), no se identificaron valores de VIF>10, por lo que también se rechaza una probable colinealidad entre los ítems. Los índices de condición muestran valores menores a 30, de esta forma se asume que existe independencia entre los ítems. 
APOYO ORGANIZACIONAL PERCIBIDO Y COMPROMISO ORGANIZACIONAL, SU RELACIÓN CON EL CLIMA ÉTICO EN ENTIDADES DE FISCALIZACIÓN SUPERIOR

Mediante la prueba de medida Kaiser-Meyer-Olkin (KMO) se demuestra que los grupos de variables son homogéneos cuanto más cerca de uno tenga el valor obtenido del test KMO, implica que la relación entre las variables es alta, de esta manera se considera adecuado un valor igual o superior a 0.70 , el cual sugiere una interrelación satisfactoria entre los ítems (Hair, Anderson, Tatham, y Black, 2010), (ver tabla 2).

Tabla 2

Prueba de KMO y test de esfericidad de Bartlet

\begin{tabular}{lcccc}
\hline \multicolumn{1}{c}{ Variables } & Medida & \multicolumn{2}{c}{ Prueba de esfericidad de Bartlett } \\
& de KMO & Chi cuadrado & Grados de libertad & Significancia \\
\hline Apoyo Organizacional Percibido & 0.881 & 864.612 & 10 & 0.000 \\
Compromiso Organizacional & 0.897 & 644.596 & 21 & 0.000 \\
Clima Ético & 0.925 & 1318.538 & 66 & 0.000 \\
\hline
\end{tabular}

Fuente: Elaboración propia con resultados obtenidos en SPSS V. 23.

Seguidamente se presentan los resultados de asociación entre los ítems y las variables del modelo teórico objeto de estudio de esta investigación, para ello se analizaron las subdimensiones de cada constructo hasta obtener un buen ajuste en cada uno de ellos Se realizaron los cálculos para determinar los indicadores de validez de constructos: la Fiabilidad Compuesta (FC) y la Varianza Media Extraída (AVE) permitieron buscar la validez convergente, (ver tabla 3).

Tabla 3

Fiabilidad Compuesta y Varianza Media Extraída de los Constructos

\begin{tabular}{lcccc}
\hline \multicolumn{1}{c}{ Constructo } & $\begin{array}{c}\text { Alfa de } \\
\text { Cronbach }\end{array}$ & $\begin{array}{c}\text { Confiabilidad } \\
\text { Compuesta (CR) }\end{array}$ & $\begin{array}{c}\text { Varianza Promedio } \\
\text { Extraída (AVE) }\end{array}$ & $\begin{array}{c}\text { Varianza Máxima } \\
\text { Compartida (MSV) }\end{array}$ \\
\hline $\begin{array}{l}\text { Compromiso } \\
\text { Organizacional }\end{array}$ & 0.911 & 0.914 & 0.681 & 0.762 \\
$\begin{array}{l}\text { Clima Ético } \\
\begin{array}{l}\text { Apoyo Organizacional } \\
\text { Percibido }\end{array}\end{array}$ & 0.856 & 0.858 & 0.468 & 0.572 \\
\hline
\end{tabular}

Fuente: Elaboración propia con resultados obtenidos en SPSS V. 23. 
APOYO ORGANIZACIONAL PERCIBIDO Y COMPROMISO ORGANIZACIONAL, SU RELACIÓN CON EL CLIMA ÉTICO EN ENTIDADES DE FISCALIZACIÓN SUPERIOR

El modelo ajustado obtenido como resultado de la investigación se muestra en la figura 3 . El modelo evidencia ajuste a los datos como se muestra en la tabla 4; por otra parte se observa la normalidad de los datos determinada a partir de los cifras de las covarianzas residuales estandarizadas contenidas en la tabla 5 y en la figura 4 para verificar que los datos tienen distribución normal con media cero (Arbuckle, 2010).

Mediante el modelo de rutas determinado se determinaron los efectos directos e indirectos que existen entre las variables observadas. Las relaciones causales apuntan hacia la comprobación de las hipótesis del estudio donde el AOP y el CO impactan al CE. Se advierte además que el AOP impacta al CO y al CE, (ver tablas 6 y 7 ).

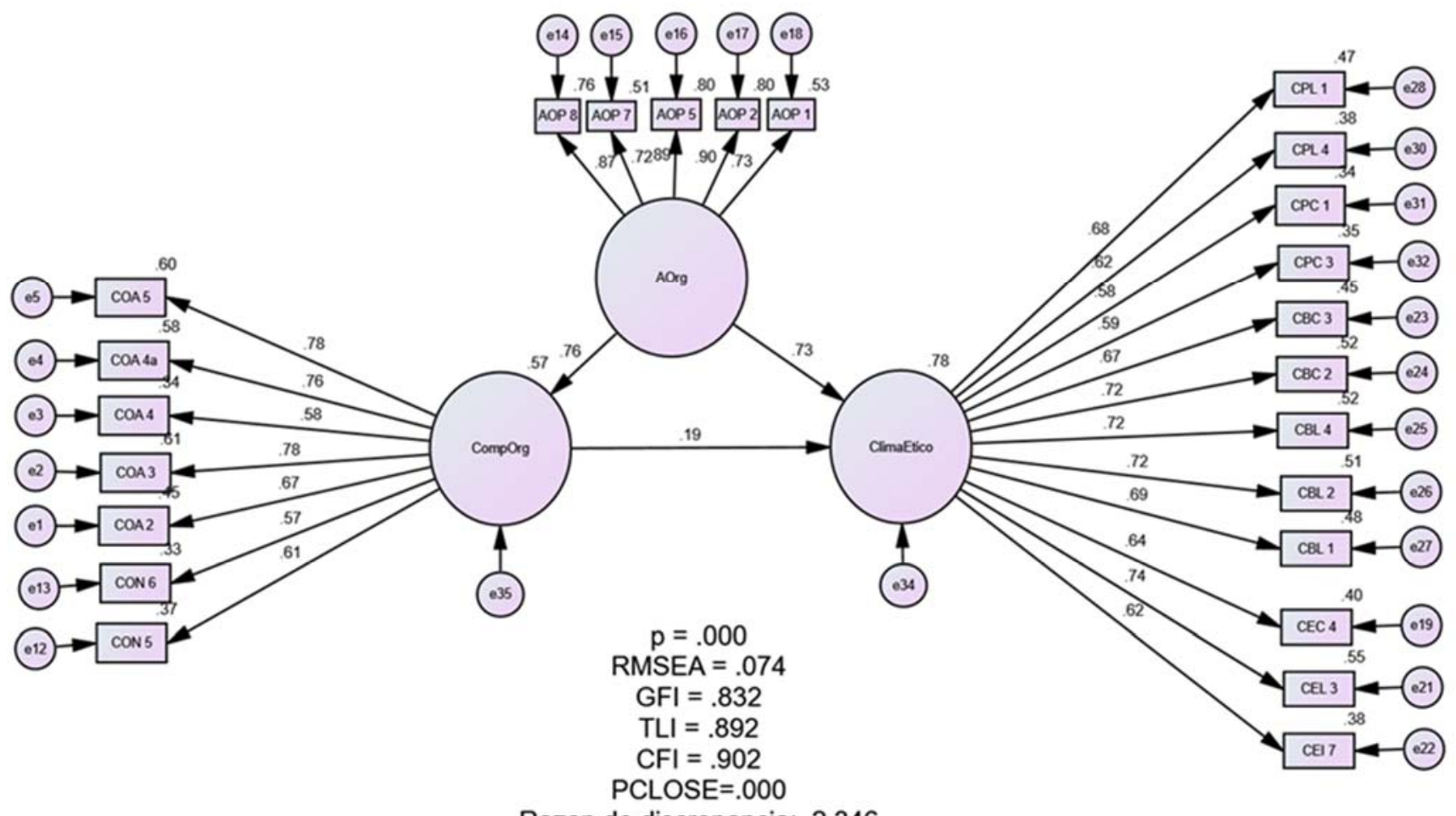

Razon de discrepancia: 2.346

\section{Figura 3. Modelo de AOP, CO y CE.}

Fuente: Elaboración propia con resultados de AMOS V. 23 con datos de la investigación. 
APOYO ORGANIZACIONAL PERCIBIDO Y COMPROMISO ORGANIZACIONAL, SU RELACIÓN CON EL CLIMA ÉTICO EN ENTIDADES DE FISCALIZACIÓN SUPERIOR

\section{Tabla 4}

Bondad de ajuste del modelo confirmado

\begin{tabular}{|c|c|c|c|}
\hline Medida de ajuste & $\begin{array}{c}\text { Valor } \\
\text { obtenido }\end{array}$ & Valor de referencia & Resultado \\
\hline \multicolumn{4}{|l|}{ Medidas de ajuste absoluto } \\
\hline Nivel de significación de x2 (Chi cuadrada) & 584.233 & $p\left(x^{2}\right)$ debe ser superior a 0.05 & Aceptable \\
\hline Razón X2/gl (CMIN DF) & 2.346 & Menor a 3 & Aceptable \\
\hline Índice de Bondad de Ajuste (GFI) & 0.832 & Mayor a 0.90 & Aceptable \\
\hline Error de Aproximación Cuadrático Medio (RMSEA) & 0.074 & Menor a 0.08 & Aceptable \\
\hline Índice de Error Cuadrático Medio (RMR) & 0.149 & Admisible a un valor cerca de 0 & Aceptable \\
\hline \multicolumn{4}{|l|}{ Medidas de ajuste incremental } \\
\hline Índice de Ajuste Comparativo (CFI) & 0.902 & Mayor a 0.95 & Aceptable \\
\hline Índice Normalizado de Ajuste (NFI) & 0.842 & Mayor a 0.90 & Aceptable \\
\hline Índice Ajustado de Bondad de Ajuste (AGFI) & 0.797 & Mayor o igual a 0.90 & Aceptable \\
\hline Índice Tucker Lewis (TLI) & 0.892 & Mayor o igual a 0.90 & Aceptable \\
\hline \multicolumn{4}{|l|}{ Medidas de ajuste de parsimonia } \\
\hline Índice de Bondad de Ajuste de Parsimonia (PCFI) & 0.814 & Entre 0.5 y 0.7 & Aceptable \\
\hline Índice de Ajuste Normado de Parsimonia (PNFI) & 0.76 & Valor cercano a 1 & Aceptable \\
\hline
\end{tabular}

Fuente: Elaboración propia con resultados de AMOS V. 23.

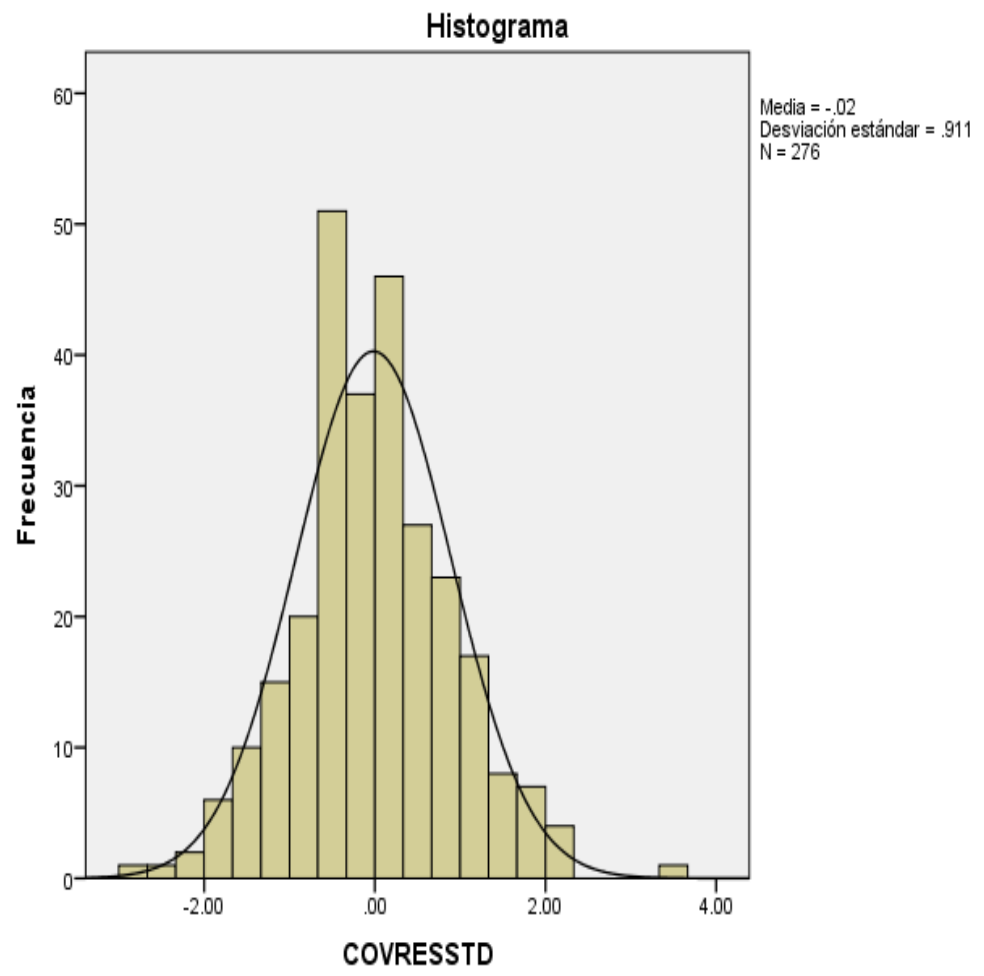

Figura 4. Histograma de distribución normal de covarianzas residuales estandarizadas al modelo propuesto.

Fuente: Elaboración propia con resultados de AMOS V. 23. 
APOYO ORGANIZACIONAL PERCIBIDO Y COMPROMISO ORGANIZACIONAL, SU RELACIÓN CON EL CLIMA ÉTICO EN ENTIDADES DE FISCALIZACIÓN SUPERIOR

\section{Tabla 5}

Estadísticas de normalidad y covarianzas residuales estandarizadas del modelo

\begin{tabular}{llc}
\hline & & COVRESSTD \\
\hline $\mathrm{N}$ & Media & 276 \\
Parámetros normales ${ }^{\mathrm{a}, \mathrm{b}}$ & Desviación estándar & -.0163 \\
& Absoluta & .91115 \\
Máximas diferencias extremas & Positivo & .048 \\
& Negativo & .040 \\
& & -.048 \\
Estadístico de prueba & & .048 \\
Sig. asintótica (bilateral) & & $.200^{\mathrm{c}, \mathrm{d}}$ \\
\hline
\end{tabular}

Nota. A) La distribución de prueba es normal. B) Se calcula a partir de datos. C) Corrección de significación de Lilliefors. D) Esto es un límite inferior de la significación verdadera.

Fuente: Elaboración propia son resultados de AMOS V. 23.

\section{Tabla 6}

Efectos directos estandarizados del Modelo de Rutas

\begin{tabular}{lcccrrr} 
& AOP & COA & CEB & CEE & CEP \\
\hline COA & 0.671 & 0 & 0 & 0 & 0 \\
CEB & 0.545 & 0.224 & 0 & 0.141 & 0 \\
CEE & 0.486 & 0 & 0 & 0 & 0.412 \\
CEP & 0 & 0 & 0.743 & 0 & 0 \\
CON & 0 & 0.649 & 0 & 0 & -0.172 \\
\hline
\end{tabular}

Fuente: Elaboración propia con resultados de AMOS V. 23. 
APOYO ORGANIZACIONAL PERCIBIDO Y COMPROMISO ORGANIZACIONAL, SU RELACIÓN CON EL CLIMA ÉTICO EN ENTIDADES DE FISCALIZACIÓN SUPERIOR

\section{Tabla 7}

Efectos indirectos estandarizados del modelo de rutas

\begin{tabular}{lrrrrr}
\hline & \multicolumn{1}{l}{ AOP } & \multicolumn{1}{l}{ COA } & \multicolumn{1}{l}{ CEB } & \multicolumn{1}{l}{ CEE } & \multicolumn{1}{l}{ CEP } \\
\hline COA & 0 & 0 & 0 & 0 & 0 \\
CEB & 0.254 & 0.01 & 0.045 & 0.006 & 0.061 \\
CEE & 0.245 & 0.072 & 0.32 & 0.045 & 0.019 \\
CEP & 0.593 & 0.174 & 0.034 & 0.11 & 0.045 \\
CON & 0.333 & -0.03 & -0.134 & -0.019 & -0.008 \\
\hline
\end{tabular}

Fuente: Elaboración propia con resultados de AMOS V. 23.

\section{CONCLUSIONES}

Se realizó la comprobación de las hipótesis planteadas para el contexto de las EFSLL, con sustento en el modelo teórico propuesto, al alcance del análisis de ecuaciones estructurales confirmatorio del modelo global de la investigación donde se ajustan los valores para precisar y confirmar los pesos de regresión estimados y estandarizados donde los indicadores muestran un CR mayor de 1.98 y P menor de 0.005, (ver tabla 6 y 7 , y figura 3).

\section{Tabla 8}

Pesos de regresión estimados y estandarizados del modelo confirmado

\begin{tabular}{cccccccc}
\hline & & & Estimados & S.E. & C.R. & P & Estandarizados \\
\hline CompOrg & $<---$ & AOrg & 0.492 & 0.056 & 8.748 & $* * *$ & 0.756 \\
ClimaEtico & $<---$ & AOrg & 0.474 & 0.057 & 8.268 & $* * *$ & 0.732 \\
ClimaEtico & $<---$ & CompOrg & 0.186 & 0.073 & 2.545 & 0.011 & 0.187 \\
\hline
\end{tabular}

Fuente: Elaboración propia con resultados del modelo en software AMOS V. 23.

Los resultados estadísticos mostrados en la tabla 4, Chi-cuadrada de 584.233, la razón de discrepancia de los grados de libertad de 2.346 permite observar un buen ajuste y que se confirma con un Root Mean Square Error of Approximation (RMSEA) de 0.074 indicando que se trata de un modelo parsimonioso. 
APOYO ORGANIZACIONAL PERCIBIDO Y COMPROMISO ORGANIZACIONAL, SU RELACIÓN CON EL CLIMA ÉTICO EN ENTIDADES DE FISCALIZACIÓN SUPERIOR

De esta forma, mediante los valores contenidos en la figura 3 y tabla 8 se tiene que: Para la hipótesis H1 "El compromiso y el Apoyo Organizacional Percibido se relacionan positivamente", el peso de regresión estandarizado de AOP tiene un efecto positivo sobre CO observando una relación positiva de 0.756 que confirma la hipótesis de estudio. En la hipótesis H2 se establece que: "El CO tiene efecto significativo en el CE de la organización", el peso de regresión estandarizado de $\mathrm{CO}$ tiene un efecto positivo sobre CE observando una relación positiva de 0.187 que confirma la hipótesis de estudio. La hipótesis H3 establece que: "El AOP tiene efecto significativo en el CE de la organización", el peso de regresión estandarizado de AOP tiene un efecto positivo sobre CE observando una relación positiva de 0.732 que confirma la hipótesis de estudio.

En el anexo 1, se observan la proporción para cada componente en la integración de sus ítems, de esta manera, se determina que CE es la dimensión mejor explicada ya que representa 0.777 , seguida de CO con una proporción de 0.572 , siendo estos dos componentes los de mayor integración, no obstante, AOP no muestra una proporción explicada por sus ítems por tratarse de un constructo unidimensional. El resultado de las cargas factoriales del modelo confirmatorio de ecuaciones estructurales permite observar a la variable AOP como el elemento que mayor interacción con los constructos CO y CE.

Conforme a la teoría del AOP, se confirma que el AOP es una variable afectiva considerada como un antecedente del $\mathrm{CO}$, esto significa que cuando se tiene un alto nivel de AOP atiende la necesidad de los individuos de pertenencia y generar incrementos del esfuerzo en favor de su organización y ser objeto de recompensas (Eisenberger, et al. 1986, Kirana, Yahva, Zaharos, Manzor y Warokka, 2012; Miao, 2011).

EI COC aunque está relacionado con la percepción de alternativas disponibles y los costos de dejar la organización, no conservó ningún ítem en el modelo final debido a que no presentó buen ajuste, este resultado es congruente con los reportados por Litllewood, H. (2003).

Por su parte, el compromiso afectivo relacionado con la identificación del empleado y su involucramiento con la institución, conservó cinco ítems con una buena carga factorial, (COA2, COA3, COA4, COA4a, COA5). Para el compromiso normativo que se refiere a la lealtad y sentido de obligación del empleado, se conservaron dos ítems (CON5, CON6), que es congruente con lo que determinaron Allen y Meyer (1990). Esta identificación, involucramiento, lealtad y sentido de 
APOYO ORGANIZACIONAL PERCIBIDO Y COMPROMISO ORGANIZACIONAL, SU RELACIÓN CON EL CLIMA ÉTICO EN ENTIDADES DE FISCALIZACIÓN SUPERIOR

obligación permiten afirmar que los servidores públicos de los EFSLL, hacen posible el funcionamiento, a pesar de la rotación de los titulares.

De acuerdo con los resultados del CO podemos interpretar que los servidores públicos de los EFSLL que conformaron la muestra, tienen moderado compromiso de lealtad con la institución y los lazos afectivos entre ellos aún están en desarrollo, la permanencia en sus trabajos no es de consideración y esto se explica por las características especializadas del trabajo que desempeñan, manifestado en la antigüedad de años de servicio que acumulan. De igual forma, se comprueba que las personas pueden sentir de manera simultánea y distinta intensidad, más de un tipo de compromiso debido a que son el resultado de las experiencias personales, donde se pueden atender a particularidades de la organización.

La investigación está restringida al contexto de las EFSLL en México y de manera específica con personal de las siete EFSLL del país que aceptaron participar, los resultados presentados son evidencia para ese contexto dentro del marco temporal en que se realizó por lo que no se busca su generalización a otros contextos o culturas; toda vez que el contexto de este trabajo lo constituyeron las EFSLL, se tienen aspectos particulares que pueden estar ausentes en el entorno laboral en otros contextos, debido a la situación particular en cada organización.

Se hace necesario realizar otros estudios para probar y perfeccionar estos instrumentos, por ello se recomienda replicar esta investigación en otras organizaciones públicas que cuenten con servicio civil de carrera en operación, para observar el comportamiento de cada una de las dimensiones. En el caso del CO y el AOP, se propone la realización de investigaciones de tipo longitudinal en instituciones de la administración pública de los tres órdenes de gobierno (federal, estatal y municipal) a fin de contrastar resultados al inicio y al final de un periodo de gestión de gobierno. 
APOYO ORGANIZACIONAL PERCIBIDO Y COMPROMISO ORGANIZACIONAL, SU RELACIÓN CON EL CLIMA ÉTICO EN ENTIDADES DE FISCALIZACIÓN SUPERIOR

\section{REFERENCIAS BIBLIOGRÁFICAS}

Arbuckle, J. (2010). IBM SPSS Amos 19 User's Guide. Chicago. Estados Unidos de América: IBM.

Allen, N. y Meyer, J. (1990). The Measurement and Antecedents of Affective, Continuance and Normative Commitment to the Organization. Journal of Occupational Psychology, 63, pp. 118. Recuperado de http://workandbabies.com/wp-content/uploads/2009/11/allen-myer1990.pdf.

Álvarez, G. (2008). Determinantes del compromiso organizacional. ¿Están los trabajadores a tiempo parciales menos comprometidos que los trabajadores a tiempo completo? Cuadernos de estudios empresariales, 18, pp. 73-88. Recuperado de http://revistas.ucm.es/index.php/CESE/article/viewFile/CESE0808110073A/9307.

Arnaud, A. y Schminke, M. (s.f.). Understanding the ethical context of organizations: the role of ethical climate, collective moral emotion, and collective ethical efficacy on ethical behavior in organizations. Proyecto de artículo. Recuperado de www.econ.upf.edu/docs/seminars/schminke.pdf.

Auditoría Superior de la Federación (2010). Fiscalización Superior en México, 185 Aniversario. México: Auditoria Superior de la Federación.

Betanzos, N. y Paz, F. (2007). Análisis psicométrico del compromiso organizacional como variable actitudinal. Anales de psicología, 23(2), pp. 207-215. Recuperado de http://revistas.um.es/analesps/article/view/22411/21691

Blome, C. y Paulraj, A. (2013). Ethical Climate and Purchasing Social Responsibility: A Benevolence Focus. Journal of Business Ethics, 116 (3), pp. 567-585. Recuperado de http://link.springer.com/article/10.1007/s10551-012-1481-5

Cullen J. B., Parboteeah K. \& Victor B. (2003). The effects of ethical climates on organizational commitment: a two-study analysis. Journal of Business Ethics; Aug 2003; 46, 127-141. Recuperado de http://link.springer.com/article/10.1023\%2FA\%3A1025089819456\#page-1

Duffy, J. A. \& Lilly, J. (2013). Do Individual Needs Moderate the Relationships between Organizational Citizenship Behavior, Organizational Trust and Perceived Organizational Support? Journal of Behavioral y Applied Management, 185-197. Recuperado de 
APOYO ORGANIZACIONAL PERCIBIDO Y COMPROMISO ORGANIZACIONAL, SU RELACIÓN CON EL CLIMA ÉTICO EN ENTIDADES DE FISCALIZACIÓN SUPERIOR

http://www.ibam.com/pubs/jbam/articles/vol14/No3/Article\%204_Duffy_\%20after\%20assist ant\%20editor.pdf

Eisenberger, R., Huntington, R., Hutchison, S. \& Sowa, D. (1986). Perceived organizational support. Journal of Applied Psychology, 71, 500-507. Recuperado de http://eisenberger.psych.udel.edu/files/22_Perceived_Organizational_Support.pdf

Eisenberger, R. (s.f.). Perceived organizationational support website. Recuperado de http://www.psychology.uh.edu/pos/default.asp

Eisenberger, R., Fasolo, P. \& Davis-LaMastro, V. (1990). Perceived organizational support and employee diligence, commitment, and innovation. Journal of Applied Psychology, 75(1), pp. 51-59. Recuperado de http://eisenberger.psych.udel.edu/files/20_Perceived_Organizational_Support_and_Emplo yee_Diligence.pdf

Eisenberger, R., Stinglhamber, F., Vandenberghe, C., Stinglhamber, F., Vandenberghe, Sucharski, I. y Rhoades, L. (2002). Perceived supervisor support: contributions to perceived organizational support and employee retention. Journal of Applied Psychology, 87(3), pp. 565-573. Recuperado de http://www.psychology.uh.edu/faculty/eisenberger/files/02_perceived_supervisor_support. pdf

Ferrato, E., y Coutnho, M. (2005). Clima ético corporativo: Una aplicación de Víctor y Cullen en las empresas del Estado de Sao Paulo. Cuaderno de difusión, pp. 137-153.

Gómez, G. y Manrique, A. (2014). Clima ético en una entidad del Estado. Diversitas: perspectivas en psicología, pp. 321-336.

González, B. y García, R. (2016). Las entidades fiscalizadoras superiores: una revisión de la literatura y líneas futuras de investigación. $1^{\text {a }}$ Reunión Internacional de Investigación en Contabilidad y Auditoría (RIICA). Memoria. Recuperado de https://riica2016.unican.es/wpcontent/uploads/2016/06/RIICA-2016-Sesiones-Paralelas-Mesa-3-Comunicaciones.pdf

Hair, J., Anderson, R., Tatham, R. y Black, W. (2010). Análisis multivariante. (5a. ed.), última reimpresión. Madrid: Pearson Prentice Hall. 
APOYO ORGANIZACIONAL PERCIBIDO Y COMPROMISO ORGANIZACIONAL, SU RELACIÓN CON EL CLIMA ÉTICO EN ENTIDADES DE FISCALIZACIÓN SUPERIOR

Homans, G. (1958). Social behavior as exchange. American Journal of Sociology, 6(63), pp. 597606.

Recuperado

de

http://web.ics.purdue.edu/ hoganr/SOC\%20602/Spring\%202014/Homans\%201958.pdf

INEGI (2018). Encuesta nacional de calidad e impacto gubernamental 2017. Aguascalientes.

Kirana, K., Zaharos, F. y Warokka, A. (2012). An empirical study on the influence of perceived organizational support on academic expatriate`s organizational commitment. Journal of Organizational Management Studies. Recuperado de http://www.ibimapublishing.com/journals/JOMS/2012/565439/565439.pdf

Litllewood, H. (2003). Metanálisis del apoyo organizacional percibido y compromiso organizacional en organizaciones mexicanas. Revista Mexicana de Psicología Ocupacional, 22(2), pp. 45-56.

Maher, N. (2004). How supreme audit institution should perform and is to be managed for external audit (Independent SAI as a Precondition for Four Pillars). Recuperado de http://unpan1.un.org/intradoc/groups/public/documents/NISPAcee/UNPAN014820.pdf

Meyer, J., Allen, N. y Smith, C. (1993). Commitment to organizations and occupations: extension and test of a three-component conceptualization. Journal of Applied Psychology (79), pp. 538-551.

Recuperado

de

http://web.b.ebscohost.com/ehost/pdfviewer/pdfviewer?vid=3ysid=b2bc4dd6-081b-469eafb5-bafbe5bf5116\%40sessionmgr114yhid=122

Meyer, J. y Allen, N. (1991). A three component conceptualization of organizational commitment.

Human Resource Management Review, 1, pp. 61-89. Recuperado de http://iag_puc_2007.msimoes.dyndns.org:10080/textos/Administracao/ADM_2825-

Organizacoes/artigos/meyer\%20e\%20allen.pdf

Miao, R. (2011). Perceived organizational support, job satisfaction, task performance and organizational citizenship behavior in China. Journal of Behavioral and Applied $\begin{array}{lll}\text { Management, } & \text { 12(2). Recuperado de }\end{array}$ http://www.ibam.com/pubs/jbam/articles/vol12/no2/2\%20-\%20Ren-Tao\%20Miao.pdf

Rankumar, V. (2007). Expanding collaboration between SAls and civil society. International Journal of Government Auditing, pp. 15-21. Recuperado de http://www.intosaijournal.org/pdf/2007_staats_award_articles.pdf 
APOYO ORGANIZACIONAL PERCIBIDO Y COMPROMISO ORGANIZACIONAL, SU RELACIÓN CON EL CLIMA ÉTICO EN ENTIDADES DE FISCALIZACIÓN SUPERIOR

Rhoades, L. y Eisenberger, R. (2002). Perceived organizational support: a review of the literature. Journal of Applied Psychology, 87, pp. 698-714. Recuperado de http://eisenberger.psych.udel.edu/files/01_Perceived_Organizational_Support.pdf

Robbins, S. y Judge, T. (2013). Comportamiento organizacional (15ª . ed.) México: Pearson.

Rousseau, D. (1989). Psychological and implied contracts in organizations. Employee Responsibilities and Rights Journal. 2, pp. 121-139. Recuperado de http://link.springer.com/article/10.1007\%2FBF01384942\#page-1

Topa, G., Moriano, J. y Morales, J. (2008). Identidad social y apoyo percibido en las organizaciones: sus efectos sobre las conductas de ciudadanía. Revista Interamericana de Psicología/Interamerican Journal of Psychology, 42(2), pp. 363-370. Recuperado de http://www.redalyc.org: http://www.redalyc.org/pdf/284/28442217.pdf

Torres, L. y Díaz, J. (2012). Compromiso organizacional: actitud asertiva para la competitividad de las organizaciones. Formación Gerencial, 11(1), pp. 80-102. Recuperado de http://revistas.luz.edu.ve/index.php/rfg/article/viewFile/11342/10975

Victor, B. y Cullen, J. B. (1987). A theory and measure of ethical climate in organizations. Research in Corporato Social Performance and Policy, (9), pp. 51-71. Recuperado de http://www.amsciepub.com/doi/pdfplus/10.2466/pr0.1993.73.2.667

Victor, B. y Cullen, J. (1988). The organizational bases of ethical work climates. Administrative Science Quarterly, 33(1), pp. 101-125. Recuperado de http://web.b.ebscohost.com/ehost/pdfviewer/pdfviewer?sid=b9cb20f7-c73e-4cb2-963ddb3be67d2c15\%40sessionmgr112yvid=0yhid=121

Webber, S. (2007). Ethical climate typology and questionnaire: a discussion of instrument modifications. The Journal of Academic Librarianship, 33(5), pp. 567-580. Recuperado de http://ac.els-cdn.com/S0099133307001255/1-s2.0-S0099133307001255main.pdf?_tid=27614cb6-2eec-11e4-9bd500000aab0f6cyacdnat=1409255433_2a75b7e5ae97ea687d21e4c545dd05f3 
APOYO ORGANIZACIONAL PERCIBIDO Y COMPROMISO ORGANIZACIONAL, SU RELACIÓN CON EL CLIMA ÉTICO EN ENTIDADES DE FISCALIZACIÓN SUPERIOR

\section{ANEXOS}

\section{Anexo 1}

Resultados del modelo AOP, CO y CE

\begin{tabular}{|c|c|c|c|}
\hline Ítems & $\begin{array}{l}\text { Carga } \\
\text { factorial }\end{array}$ & $\begin{array}{l}\text { Correlación múltiple al } \\
\text { cuadrado }\end{array}$ & $\begin{array}{l}\text { Proporción explicada } \\
\text { por sus ítems }\end{array}$ \\
\hline $\begin{array}{l}\text { AOP 7. La institución me toma en cuenta cuando } \\
\text { adopta decisiones que me afectan directamente. }\end{array}$ & 0.720 & 0.512 & \\
\hline $\begin{array}{l}\text { AOP 5. La institución me valora cuando realizo el } \\
\text { trabajo lo mejor posible. }\end{array}$ & 0.890 & 0.800 & \\
\hline $\begin{array}{l}\text { AOP 2. La institución se enorgullece de mis } \\
\text { logros en el trabajo. }\end{array}$ & 0.900 & 0.802 & \\
\hline $\begin{array}{l}\text { AOP 1. La institución valora mi contribución para } \\
\text { su bienestar. }\end{array}$ & 0.730 & 0.534 & \\
\hline $\begin{array}{l}\text { COA } 5 . \text { Me siento como parte de una familia en } \\
\text { esta institución. }\end{array}$ & 0.780 & 0.604 & \\
\hline $\begin{array}{l}\text { COA 4. Me siento emocionalmente vinculado con } \\
\text { esta institución. }\end{array}$ & 0.760 & 0.583 & \\
\hline $\begin{array}{l}\text { COA 4. Disfruto hablando de mi institución con } \\
\text { gente que no pertenece a ella. }\end{array}$ & 0.580 & 0.338 & \\
\hline $\begin{array}{l}\text { COA 3. Tengo una fuerte sensación de } \\
\text { pertenecer a mi institución. }\end{array}$ & 0.780 & 0.608 & 0.572 \\
\hline $\begin{array}{l}\text { COA 2. Realmente siento como si los problemas } \\
\text { de esta institución fueran mis propios problemas. }\end{array}$ & 0.670 & 0.445 & \\
\hline $\begin{array}{l}\text { CON 6. A esta institución le debo mucho de lo } \\
\text { que soy. }\end{array}$ & 0.570 & 0.329 & \\
\hline $\begin{array}{l}\text { CON } 5 \text {. No renunciaría a esta institución ahora, } \\
\text { porque me siento comprometido con su gente. }\end{array}$ & 0.610 & 0.366 & \\
\hline $\begin{array}{l}\mathrm{CPL} 1 \text {. Aquí es muy importante seguir las reglas } \\
\text { y procedimientos de la Institución. }\end{array}$ & 0.680 & 0.465 & \\
\hline $\begin{array}{l}\text { CPL 4. Las personas exitosas en esta Institución } \\
\text { obedecen estrictamente las políticas } \\
\text { institucionales. }\end{array}$ & 0.620 & 0.385 & \\
\hline $\begin{array}{l}\text { CPC 1. En esta Institución la principal } \\
\text { consideración al tomar una decisión es si esta } \\
\text { viola alguna ley. }\end{array}$ & 0.580 & 0.336 & 0.777 \\
\hline $\begin{array}{l}\text { CPC 3. En esta Institución se espera que las } \\
\text { personas cumplan estrictamente con las } \\
\text { disposiciones legales y normas profesionales. }\end{array}$ & 0.590 & 0.352 & \\
\hline
\end{tabular}


APOYO ORGANIZACIONAL PERCIBIDO Y COMPROMISO ORGANIZACIONAL, SU RELACIÓN CON EL CLIMA ÉTICO EN ENTIDADES DE FISCALIZACIÓN SUPERIOR

\begin{tabular}{|c|c|c|c|}
\hline $\begin{array}{l}\text { CBC 3. Las personas en esta Institución se } \\
\text { preocupan activamente por el interés de los } \\
\text { usuarios de sus servicios. }\end{array}$ & 0.670 & 0.452 & \\
\hline $\begin{array}{l}\text { CBC 2. Las personas en esta Institución tienen } \\
\text { un fuerte sentido de responsabilidad hacia la } \\
\text { comunidad externa. }\end{array}$ & 0.720 & 0.515 & \\
\hline $\begin{array}{l}\mathrm{CBL} \text { 4. Las personas en esta Institución } \\
\text { consideran importante el espíritu de equipo. }\end{array}$ & 0.720 & 0.524 & \\
\hline $\begin{array}{l}\text { CBL 2. La preocupación más importante es } \\
\text { procurar el bien de todas las personas de la } \\
\text { Institución. }\end{array}$ & 0.720 & 0.514 & \\
\hline $\begin{array}{l}\text { CBL 1. Lo más importante para nosotros es lo } \\
\text { que sea mejor para todos en la Institución. }\end{array}$ & 0.690 & 0.478 & 0.777 \\
\hline $\begin{array}{l}\text { CEC 4. En esta Institución siempre se buscan } \\
\text { decisiones eficientes a los problemas. }\end{array}$ & 0.640 & 0.404 & \\
\hline $\begin{array}{l}\text { CEL 3. Las decisiones en esta Institución son } \\
\text { evaluadas principalmente en términos de su } \\
\text { contribución al logro de los resultados. }\end{array}$ & 0.740 & 0.554 & \\
\hline $\begin{array}{l}\text { CEI 7. En esta institución se espera que cada } \\
\text { individuo sea tomado en cuenta a la hora de } \\
\text { tomar decisiones. }\end{array}$ & 0.620 & 0.385 & \\
\hline
\end{tabular}

Fuente: Elaboración propia con resultados del modelo en software AMOS V. 23. 